\title{
A Novel Approach Using a Silicone Tube to Assist Microsurgical Anastomosis Training in Animal Models
}

\author{
Yuan Dian Zheng, BS; John J. Corvi, BS; Celine F. Nicolas, BS; Yelena Akelina, DVM, MSc* \\ Department of Orthopedic Surgery, Columbia University Irving Medical Center, New York, NY, United States
}

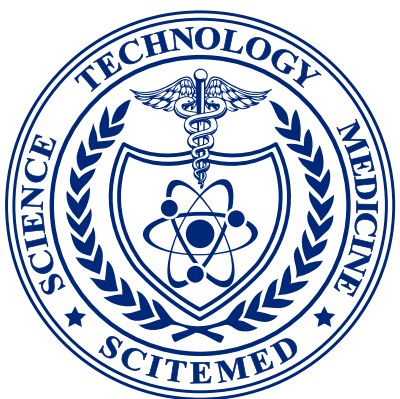

\begin{abstract}
Various reconstructive surgical procedures depend on the achievement of a patent microvascular anastomosis. Achieving clinical competency in this refined skill can begin with intensive training on live animal models. The early training stage can be stressful for novices as the rat vessels used during microvascular anastomosis training courses are often too small and too fragile for novices to optimally maintain the vascular lumen. In this paper, the authors discuss a silicone tube that provides structural support to vessels throughout the entire precarious suturing process. This modification of the conventional microvascular anastomosis technique may facilitate initial skill acquisition using the rat model.
\end{abstract}

\section{INTRODUCTION}

Microvascular anastomosis is a critical step in various subspecialties of reconstructive surgery. The creation of a patent anastomosis requires high technical skills and knowledge in suturing delicate, millimeter-scale vessels. Throughout the procedure, adequate visualization of the vessel lumen is essential as this reduces unnecessary, potentially injurious vessel manipulations and minimizes the risk of a surgeon placing suture that leads to failure. Nonliving (latex glove, silicone tubing) or living (anesthetized rat) models can be utilized to simulate clinical microvascular surgery during microsurgical training programs, including our program at the Microsurgery Training and Research Laboratory of the Columbia University Irving Medical Center. We train approximately 150 surgeons a year in basic and advanced clinical microsurgery and supermicrosurgery techniques. Our training curriculums, along with other microsurgery programs, offer individualized didactic and hands-on instruction combined with the rapid progression using low-fidelity models (surgical glove, silicone tubing) to high-fidelity models (live animals) [1].

Non-living biomodels such as the chicken leg or porcine thigh serve to bridge the progression to models that provide a more realistic simulation of living human vessels $[2,3]$. However, the discrepancies in vessel caliber and wall thickness between these models and the most commonly used microsurgery training model, the live rat, still render the transition challenging for trainees. We observe that the ability to adequately visualize the lumen and vessel edges is significantly hampered by the nature in which arterial and venous walls collapse following the occlusion of blood flow (Figure 1A). The diameter of microvessels (about $1 \mathrm{~mm}$ or less) also causes difficulties in placing forceps into the lumen. To overcome this obstacle, trainees tend to repeatedly manipulate tissue, inadvertently catch the back-wall or place uneven sutures, and inadequately oppose the vessel walls. These maneuvers may account for lower patency rates and damage the fragile vessel tissue, inevitably leading to failure and inadequate acquisition of technical skills during the limited training period [4].

We propose a novel hybrid model for training inspired by the intraluminal tubing method, previously described by Marre and Hontanilla, who inserted a plastic tube into the chicken artery through a hole to ameliorate the difficulties in training using non-living animal models [5]. Our approach incorporates a plastic tube that holds the anastomosis sites in position, thus enhancing access and visualization of the lumen to support independent completion of anastomoses in the live rat training model. This intravascular stent offers a way to compress the steep-learning curve of basic microsurgical skill acquisition and facilitate trainees during the transition from lower fidelity models to high-fidelity live animal models.

\section{PROCEDURE}

After general preparation and dissection of rat femoral or common carotid artery (about $0.8 \mathrm{~mm}$ in diameter, an approximator clamp is applied to the vessel, and a transverse transection is made at the middle portion of the vessel between the clamps. A silicone tube with an external diameter of $0.7 \mathrm{~mm}$ is then cut into a short 2-3 mm segment (Figure 1B). The edge of the tube is cut diagonally and inserted into the lumen of one end of anastomosis, and the same procedure is performed to the other end (Figure 1C). Eight stitches are then thrown through both blood vessel wall and inserted plastic tube circumferentially to complete the anastomosis (Figure 1D and 1E). The same method can be used to complete the vein as well.

\section{DISCUSSION}

Intravascular stenting has been an attractive method for aiding the intracacies surrounding microvascular anastomosis procedures. Previous stent methods for microvascular anastomosis include stents that remain intravascular for only part of the suturing procedure, along with stents that fully dissolve into the blood during the postoperative healing period. These various types of stents reportedly simplify and shorten the complex microvascular suture process, enhance patency, minimize vessel wall damage, and improve the reliability of the anastomoses performed [6]. No reports have been published on the use of silicone tubing for microvascular anastomosis training in rat models.

The intravascular plastic tube serves as a scaffold for the vessel walls. It significantly enhances visualization of the lumen. The tubing minimizes the damage from rough vessel manipulation and also reduces the chances of creating an inadvertent back-wall stitch. This method lowers the difficulty of the fundamental anastomosis exercise while preserving a level of microsurgical difficulty beyond that of the non-animal model, as the trainee must work under a restricted anatomical field and be wary of extended periods of ischemia and anesthesia while operating in a living animal. Using rat models, which 

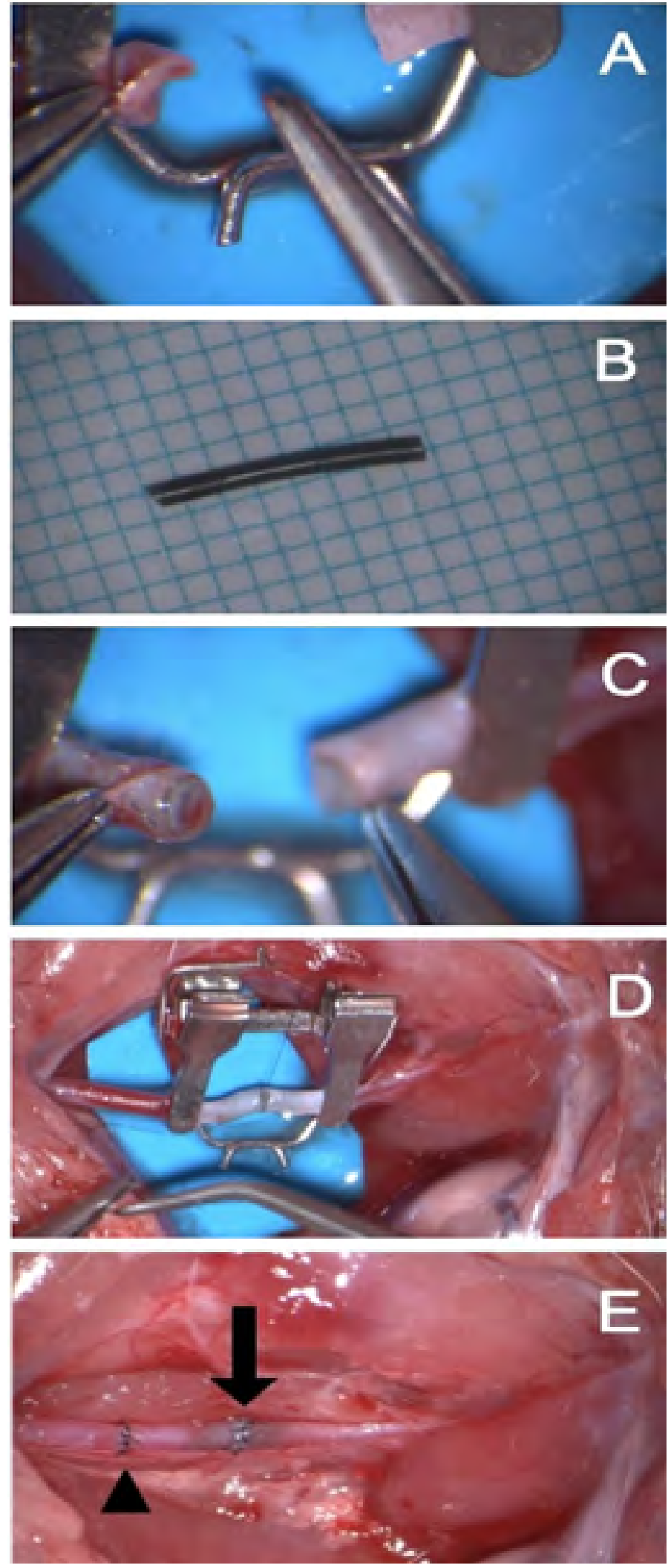

Figure 1. (A) Occlusion of blood flow through a 1-mm artery can cause the artery to collapse and lose its cylindrical shape, hampering adequate visualization of the lumen. (B) Preparation of a silicone tube with a 0.7-mm external diameter. (C) Tubes insertion into each arterial stump enhances visualization of the lumen. (D) Completion of the microarterial anastomosis with the intravascular stent. (E) The normal anastomosis (arrowhead) and plastic tubing technique (arrow). The artery is patent. imitate the relationship of blood flow, tissue response, and time in human vessels, is valuable to the trainee as well. A previous study done by Wei FC, et al. showed that the insertion of a stent or silicone tube may not produce more complications to the intima than the conventional dilatation manipulation with forceps [7]. Thus, we believe this novel exercise can help students more easily visualize the lumen, avoid back wall stitches, and ultimately, build their confidence to progressively advance from non-living to living animal models with small size blood vessels.

\section{CONCLUSION}

The authors note that this method should only be served as an intermediate step for microsurgery trainees who have difficulty in transitioning from non-living to living model during an intensive, fast-paced training curriculum.Using our proposed model, patency was evident in Video 1 (https://doi. org/10.24983/scitemed.imj.2020.00122).

\section{ARTICLE INFORMATION}

*Correspondence: *Yelena Akelina, DVM, MSc. Microsurgery Research and Training Laboratory, Columbia University. 622 West 168th Street, PH 1158, New York, NY, 10032, United States. Email: ya67@cumc.columbia.edu

Yuan Dian Zheng and John J. Corvi contributed equally to this work.

Received: Sept. 05, 2019; Accepted: Nov. 24, 2019; Published:Jan. 20, 2020

DOI: $10.24983 /$ scitemed.imj.2020.00122

Ethics Approval and Consent to Participate: The study is in accordance with the ethical standards of the 1964 Helsinki declaration and its later amendments or comparable ethical standards.

Funding: The study did not receive any specific grant from funding agencies in the public, commercial, or not-for-profit sectors.

Conflict of Interest: The authors report no financial or other conflict of interest relevant to this article, which is the intellectual property of the authors.

Copyright @ 2020 The Authors. This is an open-access article distributed under the terms of the Creative Commons Attribution 4.0 International License (CC-BY).

\section{REFERENCES}

1. Shurey S, Akelina Y, Legagneux J, Malzone G, Jiga L, Ghanem AM. The rat model in microsurgery education: classical exercises and new horizons. Arch Plast Surg 2014;41(3):201-218.

2. Hino A. Training in microvascular surgery using a chicken wing artery. Neurosurgery 2003;52(6):1495-1498.

3. Nam SM, Shin HS, Kim YB, Park ES, Choi CY. Microsurgical training with porcine thigh infusion model. J Reconstr Microsurg 2013;29(5):303-306.

4. Stone JP, Doherty CC, Schrag CH. Incidence and type of errors in microsurgical technique in surgical trainees. J Reconstr Microsurg 2016;32(7):528532.

5. Marre D, Hontanilla B. Intraluminal latex tubing for microsurgical training. J Reconstr Microsurg 2011;27(7):449-450.

6. Tashiro K, Yamashita S, Narushima M, Koshima I, Miyamoto S. Hemi-intravascular stenting for supermicrosurgical anastomosis. Plast Reconstr Surg Glob Open 2017;5(11):e1533.

7. Wei FC, Mancer K, Zuker RM. The temporary stent technique: an easier method of micro-venous anastomosis. Br J Plast Surg 1982;35(1):92-95. 